Illinois State University

ISU ReD: Research and eData

Theses and Dissertations

$5-7-2020$

\title{
Characterizing The Transport Of Parasitoid Wasp Genaspis Hookeri Venom Serca Into Host Drosophila Hemocytes
}

Christopher Lark

Illinois State University, lark.chris84@gmail.com

Follow this and additional works at: https://ir.library.illinoisstate.edu/etd

Part of the Biology Commons

\section{Recommended Citation}

Lark, Christopher, "Characterizing The Transport Of Parasitoid Wasp Genaspis Hookeri Venom Serca Into Host Drosophila Hemocytes" (2020). Theses and Dissertations. 1386.

https://ir.library.illinoisstate.edu/etd/1386

This Thesis is brought to you for free and open access by ISU ReD: Research and eData. It has been accepted for inclusion in Theses and Dissertations by an authorized administrator of ISU ReD: Research and eData. For more information, please contact ISUReD@ilstu.edu. 


\section{CHARACTERIZING THE TRANSPORT OF PARASITOID WASP GENASPIS HOOKERI VENOM SERCA INTO HOST DROSOPHILA HEMOCYTES}

\section{CHRISTOPHER LARK}

\section{Pages}

The female endoparasitoid wasps introduce venom accompanied by an egg into the larval hosts' body cavity during oviposition. The venom contains a mixture of proteins that exploit the host’s biological processes, such as, regulating gene expression, disrupting immune signaling pathways, or inducing immune cell death to give a decided advantage to the incipient wasp to develop unchallenged. Drosophila possesses a highly conserved innate immune response that recognizes and neutralizes foreign microbes and macroparasites. The venom of Ganaspis hookeri (strain G1) endoparasitoid wasp has co-evolved to suppresses the humoral phase Drosophila's innate immune response by inhibiting $\mathrm{Ca}^{2+}$ bursts needed to induce an immune response signaling pathway. The intracellular $\mathrm{Ca}^{2+}$ concentration of host plasmatocytes is altered via a venom specific homolog of the Sarco/endoplasmic reticulum calcium ATPase (SERCA) as a virulence factor. The venom SERCA (vSERCA) is able to significantly alter host intracellular $\mathrm{Ca}^{2+}$ homeostasis, disrupting cell signaling. SERCA is a multipass transmembrane protein, suggesting that vSERCA is likely packaged for transport through the venom into host plasmatocytes. Extracellular vesicles (EVs), or venosomes, to transport a variety of venom proteins and peptides. An excellent question is how vSERCA makes its way from the venom glands of G1 and into host plasmatocytes to affect $\mathrm{Ca}^{+2}$ levels. Here I will explore how G1 uses venosomes to package vSERCA and other key virulence factors for transport from the venom 
gland, where they are produced, into host hemocytes. I characterize how G1 venom transports vSERCA and other important virulence factors necessary for regulating host signaling pathways essential for the Drosophila larval immune response, and the underlying biogenic mechanisms of venosome directed protein transport. These results are relevant to our understanding of the evolution of hymenopteran and related species.

KEYWORDS: Ganaspis hookeri, endoparasitoid, venom, SERCA, extracellular vesicle 
CHARACTERIZING THE TRANSPORT OF PARASITOID WASP GENASPIS HOOKERI VENOM SERCA INTO HOST DROSOPHILA HEMOCYTES

CHRISTOPHER LARK

\begin{abstract}
A Thesis Submitted in Partial Fulfillment of the Requirements for the Degree of

MASTER OF SCIENCE

School of Biological Sciences

ILLINOIS STATE UNIVERSITY
\end{abstract}

2021 
(C) 2021 Christopher Lark 
CHARACTERIZING THE TRANSPORT OF PARASITOID WASP GENASPIS HOOKERI VENOM SERCA INTO HOST DROSOPHILA HEMOCYTES

CHRISTOPHER LARK

COMMITTEE MEMBERS:

Nathan Mortimer, Chair

Tom Hammond

Ben Sadd 


\section{ACKNOWLEDGMENTS}

I’d like thank James A Mobley and Kyoko Kojima for performing the mass spectrometry analysis at the UAB \& CCC Mass Spectrometry \& Proteomics Shared Facility at the School of Medicine, University of Alabama Birmingham. I'd also like to thank Nate and Alysia Mortimer for their guidance and mentorship, as well as with the other members of the Mortimer lab, making my lab experience enjoyable, educational, and productive.

C. L. 


\section{CONTENTS}

Page

ACKNOWLEDGMENTS

TABLES IV

FIGURES

CHAPTER I: INTRODUCTION 1

CHAPTER II: RESULTS

Venom Protein Analysis $\quad 5$

$\begin{array}{ll}\text { Mass Spectrometry } & 7\end{array}$

SERCA Activity Assay $\quad 9$

$\begin{array}{ll}\text { Immunohistochemistry and Imaging } & 9\end{array}$

CHAPTER III: DISCUSSION

CHAPTER IV: MATERIALS AND METHODS 17

$\begin{array}{ll}\text { Biological Material } & 17\end{array}$

$\begin{array}{ll}\text { Wasp Infections } & 17\end{array}$

$\begin{array}{ll}\text { Venom Protein Isolation } & 17\end{array}$

$\begin{array}{ll}\text { Venom Protein Purification } & 17\end{array}$

$\begin{array}{ll}\text { Venom Sonification } & 18\end{array}$

$\begin{array}{ll}\text { SDS-PAGE Gel Electrophoresis } & 18\end{array}$

$\begin{array}{ll}\text { Protein Stain } & 18\end{array}$

$\begin{array}{ll}\text { Western Blot } & 18\end{array}$

Sample Preparation for MS Analysis $\quad 19$

Mass Spec. nLC-ESI-MS2 Analysis and Database Searches 19 
Mass Spec. Peptide Filtering, Grouping, and Quantification

Ex vivo SERCA Activity Assay

Statistics

Immunohistochemistry

Bioinformatics

REFERENCES 


\section{TABLES}

Table

Page

1. Mass Spectrometry Fraction Quantification

2. Mass Spectrometry Analysis Fraction 5 Preview

3. Biological Process GO Terms

4. Cell Composition GO Terms

5. Molecular Function GO Terms 


\section{FIGURES}

Figure $\quad$ Page

1. Sucrose Gradient 23

2. Protein Gel and Western Blots 24

3. Venom Fractions 1-5 Calcium Assay Statistic Plots 25

4. G1 Venom Apparatus Confocal Images 26

5. G1 Venom Gland Confocal Images $\quad 27$ 


\section{CHAPTER I: INTRODUCTION}

Hymenoptera is a diverse order of insects that includes bees, sawflies, ants, and wasps. ${ }^{1-4}$ Parasitoid wasps make up a large superfamily of hymenopterans and obligately infect other arthropod species to allow for the development of their offspring. Female parasitoids use an ovipositor to lay their eggs during infection of their host and can be classified as ectoparasitoids that oviposit an egg onto the host, or endoparasitoids which infect the host by injecting an egg into its body cavity (hemocoel). ${ }^{1,4-10}$

The host hemocoel is a hostile environment for the developing endoparasitoid wasp egg, with hosts mounting a range of innate immune responses, both cellular and humoral, that are intended to kill the egg or arrest its development. ${ }^{1,7-9,11}$ In response, endoparasitoids have evolved venom virulence factors that allow them to inhibit these defense responses by targeting host immune cells, or hemocytes, and preventing their function. The venom is introduced into the host along with the egg during infection and can exploit the host by modulating a variety of biological processes including regulating gene expression, disrupting hemocyte signaling pathways, or causing cell death of hemocytes, giving the advantage to the incipient wasp to develop unchallenged. ${ }^{1-4}$ Species of endoparasitoid wasps have evolved diverse strategies to facilitate infection using a variety of virulence factors. ${ }^{1,5-10}$

Venom sequencing is an emerging area of research, and understanding the evolution and function of venom proteins is an exciting and significant field. Understanding the underlying mechanisms behind how venom factors are packaged and transported can provide a specialized perspective of interspecies coevolution, and a unique perspective into protein biogenesis and transport with implications for therapeutic applications. To develop a greater understanding of the composition and function of endoparasitoid venoms, it is essential to study their activity in 
the host environment. The fruit fly, Drosophila melanogaster, is well established as a model system for studying signaling pathways that are conserved across species. D. melanogaster is infected by many species of parasitoid wasps and thus serves as an excellent model organism to explore the molecular mechanisms of parasitoid venom virulence. ${ }^{1,7-9,12}$

D. melanogaster uses a highly conserved innate immune system to recognize and respond to foreign microbes and macroparasites. ${ }^{1,7-9}$ Flies mount two types of innate immune responses: the humoral response and cellular response. The humoral response is mainly targeted against invading microbial pathogens. ${ }^{7-10}$ After entering the host's hemocoel, the space between the outer cuticle and inner organs, these pathogens first encounter the humoral immune response in the form of antimicrobial peptides (AMPs). AMPs are naturally occurring peptides that possess a broad range of antimicrobial activities. ${ }^{8,13}$ Humoral factors are also involved in the cascade of enzymes that regulate melanization activity in the hemolymph.,8,13 Following the humoral response, the cellular immune response can be activated to neutralize pathogens via phagocytosis or encapsulation. ${ }^{6-10,13}$ Due to hemocyte function often relying on humoral molecules to begin an immune activity, there is an overlap of humoral and cellular immunity. ${ }^{6-8}$

Encapsulation is a defense reaction that is activated against foreign bodies that are too large for phagocytosis, such as endoparasitoid wasp eggs and other macroparasites. ${ }^{6-8,13}$ Instead of ingesting the foreign body, a number of specialized immune cells surround and adhere to the surface of the invading macroparasite to form a continuous layer. These specialized immune cells deposit melanin which hardens into a solid capsule to seal the pathogen, resulting in death. $1,7,8,10$

For successful parasitization and development of wasp larvae, endoparasitoids that infect D. melanogaster have evolved to modify and suppress the fly’s immune response via venom 
virulence factors. The venoms of different endoparasitoid species use a wide range of mechanisms to suppress the host immune response including causing the death of host immune cells, detoxifying the host's hemocoel and inhibiting host immune pathways. ${ }^{1,6-8}$ The endoparasitoid Ganaspis hookeri (strain G1) uses the latter of these venom strategies. G1 utilizes a venom specific homolog of the Sarco/endoplasmic reticulum calcium ATPase (SERCA) as a virulence factor to inhibit host calcium (Ca2+) signaling. ${ }^{7,8}$

$\mathrm{Ca} 2+$ is a second messenger found ubiquitously across cell types and is integral to cell signaling. $1^{2,14,15}$ Physical interactions with Ca2+ can regulate the conformation and charge of a subset of signaling proteins, which in turn alters cellular activity. In both insect and human cells, the cycling of $\mathrm{Ca} 2+$ levels in the cytoplasm is regulated by calcium-induced calcium release channels for $\mathrm{Ca} 2+$ release from intracellular stores, and SERCA for Ca2+ uptake. Typically, naïve circulating immune cells maintain a low cytoplasmic concentration of Ca2+. ${ }^{10} \mathrm{After}$ contact with a pathogen, immune receptors trigger an influx of calcium to activate immune signaling, like flipping a switch that turns on the lights in a room. When this calcium influx is genetically blocked, the cellular encapsulation response is inhibited., ${ }^{7,8,10}$

Several isoforms of the SERCA gene can be found in both insect and mammalian genomes, with most organisms encoding at least two isoforms. ${ }^{7,8,16-19}$ Each isoform varies in its C-terminus, which affects the isoform's affinity for Ca2+. ${ }^{7,8}$ The venom SERCA (vSERCA), which is found only in the abdomen of the wasp, represents a highly active isoform with a high affinity for $\mathrm{Ca} 2+$ and the ability to significantly alter intracellular Ca2+ homeostasis. ${ }^{1,7}$ Following infection, vSERCA specifically targets host plasmatocytes, a hemocyte subtype that circulates in the hemolymph of fly larvae. Plasmatocytes rely on a burst of calcium to activate the cellular immune response, and vSERCA disrupts this intracellular calcium regulation in host 
plasmatocytes. ${ }^{7,8}$ This, in turn, prevents the encapsulation of the wasp egg, allowing for successful parasitization.

SERCA is a multipass transmembrane protein, ${ }^{7,8}$ suggesting that vSERCA is unlikely to be freely secreted into the aqueous host environment, but instead may be packaged for transport through the venom. It has already been established that the venoms of endoparasitoids from the Ganaspis and Leptopilina genera contain extracellular vesicles (EVs) to transport a variety of venom proteins and peptides. ${ }^{5,6,10,13} \mathrm{EVs}$ are heterogeneous structures, which are bound by a lipid membrane and are shed from the cell's plasma membrane bilayer. ${ }^{20-23} \mathrm{EVs}$ can play a major role in altering a cell's internal environment and regulating cell signaling and cell motility., 5,22 Understanding the underlying biogenic mechanisms of protein trafficking directed by EVs has made research focused on EVs a hot topic as they present a variety of potential methods of therapeutic treatments.

One outstanding question is how vSERCA makes its way from the venom glands of G1 and into host plasmatocytes to affect $\mathrm{Ca}+2$ levels. The system of delivering cell-specific packages of virulent proteins to regulate signaling pathways that occurs between endoparasitoids and their hosts is exciting but poorly understood. One of my goals is to begin understanding how the vSERCA venosomes are produced and to identify potential cargos that are necessary for regulating host cell function. My hypothesis is that G1 uses venom EVs (venosomes ${ }^{10}$ ) to package vSERCA and other key virulence factors for transport from the venom gland, where they are produced, into host hemocytes. The goal of my experiments is to test whether G1 uses venosomes as a method of transporting vSERCA and other important virulence factors necessary for regulating host signaling pathways essential for the Drosophila larval immune response. 


\section{CHAPTER II: RESULTS}

\section{Venom Protein Analysis}

To test the hypothesis that vSERCA is being transported through the venom via a venosome, a sucrose gradient (Fig. 1) was used in combination with centrifugation to separate venom components by density. Venosomes tend to be denser than individual proteins, so I used this separation to test which fraction vSERCA was found in. I would expect to find individual peptides and proteins in the top layers of the sucrose gradient (i.e. fraction 1), and I would expect to find larger complexes, such as venosomes, in the lower portion of the gradient (i.e. fraction 5).

I first wanted to use sodium dodecyl sulfate-polyacrylamide gel electrophoresis (SDSPAGE) to get an idea of the amount of protein in the venom sample, as well as to see how the proteins are distributed across the fractions. As previously mentioned, venosomes are larger complexes containing a variety of lipids and proteins. If G1 venom contains venosomes as venom transport factors, then I'd expect to see proteins in the lower (denser) fractions. I might also expect to see some venosome overlap from the fraction containing the largest quantity of vSERCA with the neighboring fractions because I wouldn't expect the sucrose gradient to perfectly isolate all of the vSERCA and subsequently all the venosomes containing vSERCA in one specific fraction.

To view proteins using SDS-PAGE, the proteins are denatured prior to being run through the gel matrix. However, it is likely that the charged lipid membrane of the venosomes would be resistant to the protein denaturation protocol and would instead need to be disrupted in a way that would allow their contents to be released. By substituting the typical sample preparation with a modified sonication protocol designed to disrupt venosomes, I expect to see a change in the result of the protein gel. 
Once the fractions are run through an SDS-PAGE gel and transferred, the lower fractions have a marked number of bands. This suggests that the proteins are not likely to be separating by size but instead separating by density and that a number of dense complexes such as venosomes are present. Additionally, there is a noticeable difference in the results when comparing unsonicated samples (Fig. 2a) to sonicated samples (Fig. 2b). There is a clear increase in the number of bands (proteins) and in some cases a change in the intensity of the signal found in each fraction in the sonicated samples compared to the unsonicated samples. This difference is likely the result of the disruption of the charged lipid membrane, supporting the idea that many G1 venom proteins are transported in venosomes. There is also some lane distortion in Fig. 2b that is likely the result of the released lipids.

Next, I performed a Western blot with an anti-SERCA antibody to determine which fraction(s) vSERCA is found in. In the Western blot (Fig. 2c) a clear signal can be seen in lanes 2, 4, and 5 between $\sim 100 \mathrm{kD}$ and $\sim 150 \mathrm{kD}$. This is the expected size of vSERCA based on previous work8. Lanes 2, 4, and 5 also show signals at $\sim 50 \mathrm{kD}$ and $\sim 100 \mathrm{kD}$. It is possible that the $\sim 100 \mathrm{kD}$ signal may be vSERCA on its own without any venosome associated complexes or post-translational modifications and has been previously observed in G1 venom. ${ }^{8}$ The $\sim 50 \mathrm{kD}$ band is unexpected and may represent another isoform or cleavage product of SERCA not yet characterized. The signal from lanes 4 and 5 is likely showing the presence of vSERCA in a large complex such as a venosome. As previously mentioned, the perfect isolation of SERCA in any form, venom or otherwise, is unlikely given the protocol. I hypothesize that the vSERCA observed in fraction 2 likely represents vSERCA that was either not yet packaged at the time of venom collection, or was lost from venosomes that were disrupted during venom collection. 
The streaking present throughout lanes 2-5 may be caused by either a high sample protein load or a large number of lipids. The amount of protein is unlikely to be the issue since I loaded the minimum amount necessary to ensure a consistently clear signal, so my prediction is that the lipid content caused this streaking. Lipids, which form the membrane of organelles like the endoplasmic reticulum or venosomes, have a polar (charged) hydrophilic head and a pair of nonpolar (uncharged) hydrophobic tails. The charged nature of lipids has been associated with disrupting sample movement in gel electrophoresis and disrupting the ability of molecular recognition assays such as mass spectrometry. The vertical streaking of the Western blot combined with the lane distortion in the protein gel suggests that the sonication of the venom fractions was enough to disrupt the lipid membranes of venosomes.

\section{Mass Spectrometry}

If vSERCA is being transported through venom as cargo inside venosomes, then it stands to reason that there may be other conserved proteins transported with vSERCA. These would likely include proteins that are essential for the proper function of vSERCA, proteins that may have virulence functions independent of vSERCA, and proteins that may play a structurally important role in venosomes. I wanted to identify these other potential proteins found in the vSERCA containing fractions to explore the possible mechanisms for the formation and function of virulent venosomes. To identify the proteins that co-localize with the vSERCA containing fraction, I submitted the fractions for mass spectrometry (MS). MS accurately measures the mass of peptides derived from the submitted fractions and maps them onto an established database of G1 proteins, and so can help me to identify what proteins are found in G1 venom, and more importantly, what proteins are co-localizing with SERCA. 
Results from our MS analysis of unsonicated venom revealed a high concentration of charged lipids, supporting the idea that venosomes are present in venom fractions. If sonication allows the cargo to be released from association with charged lipids, I expect to find a variety of proteins represented in the MS analysis of sonicated venom fractions, including vSERCA, receptor proteins that may play important roles in immunity, and venosome structural proteins.

MS data demonstrated that there were a combined 521 proteins and peptides identified across fractions 1 through 5, with each fraction having between 230-320 proteins and peptides. Fraction 5, which is where the majority of vSERCA was identified, contained 290 other proteins. I next wanted to investigate the potential roles of this other potential venosome or cargo proteins, through functional annotation. Since G1 proteins have not been annotated, I mapped them onto their D. melanogaster homologs. Homologs of all but one of the 290 proteins found in fraction 5 were identified and used to annotate the venom proteins. Specifically, I used gene ontology (GO) term mapping to identify the cellular component, molecular function, and biological process associated with the proteins identified in fraction 5. Many of the proteins found in fraction 5 are homologous to proteins that serve as structural proteins, have ion binding domains, and are associated with functioning in metabolic and catabolic pathways. Cell component and molecular function analyses supported the identified pathways; for instance, the biological process term GO:0051238 identifies a pathway that sequesters metal ions (Table 3), while the cellular component term GO:0008043 (Table 4) suggests an intracellular ferritin complex, and the molecular function term GO:0008199 (Table 5) suggests proteins with ferric iron-binding ability. 


\section{SERCA Activity Assay}

To verify that fraction 5 contains all of the proteins/complexes necessary to manipulate host Ca2+ signaling, I separately treated isolated D. melanogaster plasmatocytes with each venom fraction and sucrose control and assayed for changes in $\mathrm{Ca} 2+$ concentration. Plasmatocytes were incubated with the fluorescent Ca2+ sensor fluo-4 along with each fraction or control. Fluo-4 is a dosage-sensitive $\mathrm{Ca} 2+$ sensor, meaning that fluo-4 fluorescence intensity can be used as a readout of cytoplasmic Ca2+ levels24. I would expect to see a change in fluo-4 fluorescence intensity when the cells were treated with a fraction that modifies cytoplasmic $\mathrm{Ca} 2+$.

Plasmatocytes from each treatment were read in a fluorescent cytometer to measure fluo4 fluorescence intensity. Fraction 5, which contained vSERCA, caused a marked decrease in fluorescence intensity relative to control (a decrease of 150 units of fluorescent intensity, Fig. 3), whereas the other fractions had only small effects on plasmatocyte Ca2+ concentration.

\section{Immunohistochemistry and Imaging}

A transmembrane protein such as SERCA does not function as a freely circulating molecule but instead resides within a membrane, specifically the sarcoplasmic or endoplasmic reticulum in the case of SERCA. SERCA is found in nearly all tissues and is particularly abundant in muscle, therefore, I would expect to find SERCA localized in muscle tissue of G1, including muscle found in the venom apparatus. I would also expect to find vSERCA localized inside the venom gland, where virulence factors would be transcribed, translated and packaged into venosomes, and the venom reservoir, where venom proteins are stored prior to infection. Although the tissue types and SERCA isoforms may vary in function, I expect similar patterns of localization in all of the tissues SERCA is present in. This expectation is based on what the 
known shape of sarcoplasmic and endoplasmic reticulums generally have with a series of closely branching lumens. SERCA would be located within these branching lumen membranes. ${ }^{25}$

A SERCA antibody, which was predicted to recognize all SERCA isoforms, was used to identify where SERCA is localizing in the venom apparatus. I could then predict where vSERCA is being packaged for release into the venom. Within the venom apparatus, venom proteins are produced in the venom gland, secreted into the secretory duct through which they travel for storage in the venom reservoir, ready for injection into the host during infection. Therefore, I focused my imaging on the venom gland and venom reservoir to understand vSERCA localization. Due to the SERCA being a highly conserved protein typically found in myocytes, I imaged SERCA in the muscle surrounding the venom reservoir for comparison.

In the muscle tissue, SERCA is localized in a tight pattern within the striated cells as would be expected for a sarcoplasmic reticulum localized protein. Within the venom gland, vSERCA localizes in a similar pattern to that of the muscle tissue, suggesting that it is largely localized to the endoplasmic reticulum in this tissue. However, there are some notable differences in vSERCA staining. First, the staining within the venom gland ER is much more punctate and there are discrete cytoplasmic foci, suggesting that vSERCA may be exported from the ER. These puncta are more numerous near the secretory duct suggesting they may be transported out of the gland. Finally, there is an area of diffuse vSERCA staining at the proximal entrance to the venom reservoir, suggesting that vSERCA that has already been packaged is stored in this tissue. 


\section{CHAPTER III: DISCUSSION}

The venoms of endoparasitoid wasps have evolved to enable the wasp to either disable the host's immune response or allow the invading egg to avoid activating the host immune

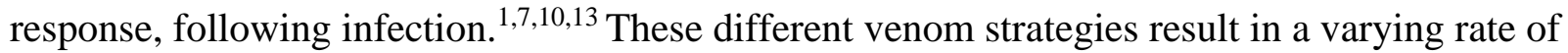
successful parasitization. Ganaspis hookeri deploys the avoidance strategy by disabling the Ca2+ intracellular signaling pathway in circulating plasmatocytes. G1 uses venom Sarco/endoplasmic reticulum ATPase (vSERCA), a venom specific isoform of a well-conserved Ca2+ ion pump, to deregulate intracellular $\mathrm{Ca}+2$ signaling in plasmatocytes. My findings support the idea that $\mathrm{G} 1$ uses venosomes to deliver virulence factors such as vSERCA to regulate the host immune response. These results lead to a model in which vSERCA is expressed in venom gland tissues where it is packaged into venosomes to be transported in the venom to target host plasmatocytes. I predict that following infection the venosome will fuse with the host cell's surface membrane and empty its cargo into the host cell, which would also allow transmembrane proteins, like vSERCA, to be incorporated into the membrane of the host cell. I would also expect to find other proteins and/or peptides in the cargo that may support vSERCA in its function or could disrupt intracellular signaling and/or metabolism.

As a starting point, G1 venom was purified and separated across five sucrose fractions to determine if vSERCA is being transported through the venom in venosomes. My Western blot results identified vSERCA in the densest fractions, suggesting that it is found in some sort of venosome or protein complex. I then used MS to identify any other well-conserved signalregulating proteins that are co-localizing with vSERCA. An initial attempt to have the purified venom fractions analyzed with mass spectrometry was not successful because of an abundance of heavily charged lipids. The abundance of charged lipids supports the idea that venosomes are 
present. Sonicating the fractions helped to release more venom proteins, which was evident in the increased number of bands present in the protein gel.

MS data demonstrated that vSERCA along with 290 other proteins were isolated in fraction 5, confirming the Western blot data showing vSERCA presence in this fraction. Fraction 5 also caused the most significant decrease in Ca2+ levels in the fluo-4 fluorescent assay. There were SERCA signal bands in lane 4 of the Western blot, which I attribute to some residual venosomes that hadn’t finished traveling through the sucrose gradient during centrifugation. A signal is also present in lane 2 of the Western blot and may be the result of vSERCA that is free of any venosomes, and may have arisen from venosomes that lysed during one of the steps of the purification process prior to the fractions being separated and sonicated.

Drosophila homologs of all but one of the 291 proteins found in fraction 5 were identified and annotated. To identify the possible functions of the proteins that co-localize with vSERCA, I used gene ontology (GO). Many of the proteins found in fraction 5 are homologous to structural proteins, ion binding proteins, and are associated with metabolic and catabolic pathways. Cell composition and molecular function supported the identified pathways. The results of the GO terms query present a pattern of functional proteins found in the vSERCA venom fraction that can be categorized as contractile filaments such as myosin filaments which can be used for transport of intercellular cargo, metabolic components like nucleotide-binding proteins that can bind ATP and GTP, catabolic components like proteasome core complexes that have the ability to degrade proteins ${ }^{26-28}$, binding metal ions, like ferric ion binding proteins to be used as nutrients for cells ${ }^{19,29}$; and signaling, such as Toll-7, which plays a role in activating the innate immune response $\mathrm{e}^{11,12,28,29}$. I predict that these cellular pathways would be affected by the proteins found in fraction 5 following infection. 
G1 venom doesn’t destroy host plasmatocytes, it only prevents them from activating the cellular immune response by blocking Ca2+ signaling, ${ }^{8,15}$. However, this most likely isn't the only change that takes place within the plasmatocytes, and it's not the only physiological change that takes place post-infection. Previous work has shown that once infected, the amount of time prior to pupation is markedly shortened, suggesting metabolic pathways are modified ${ }^{1-4}$. It is interesting that at least one type of G1 venosome essentially hijacks the hemocytes responsible for recognizing infection and signaling the timely immune response to neutralize the venom and wasp egg. In this way, G1 venom prevents the potential life-saving signal from being sent, leaving the host's existing cellular machinery to be held hostage. The plasmatocyte is like the patrolling security guard taken hostage by a vSERCA venosome and is tied up and prevented from sounding the alarm, while a crew of system hackers come in and take control of an otherwise secured network.

Thinking about the host-parasite relationship, the parasite ideally wants its host to be as healthy and robust as possible. The host condition is vital to the survival of the developing wasp. The protein Pyruvate dehydrogenase E1 alpha (Pdha) is linked to pyruvate activity ${ }^{30,31}$, which is present in fraction 5 and is involved in sucrose synthesis. It is well established that sucrose is an essential metabolic molecule ${ }^{32-35}$. By increasing the metabolism, the rate of host development also increases allowing the host to grow at an accelerated rate, allowing the developing wasp to maximize vital resources for its own development. If it helps, think about the witch in Hansel and Gretel, and how she wanted to fatten the children up before she ate them. In my scenario, the fly larvae represent the children, and the wasp larvae are the witch. Increasing the fly larvae's growth output requires an increase in energy input at the cellular level and provides increased benefit for the developing wasp. 
Cells use components such as actin filaments like capulet (capt) (Table 2) and myosin molecules like mhc (Table 2) in a way similar to a railroad for intracellular molecular transport, where actin acts as the transport rail and myosin the transport vehicle. The protein capt is an actin-binding protein that regulates actin filament assembly, and the protein mhc is a motor protein that provides contractile force along actin filaments ${ }^{36}$. Regulating contractile filament associated proteins may change the volume of intracellular molecules being transported throughout the cell to reflect the altered metabolism. With the cell doing all this extra work, it would undoubtedly need to increase nutrient intake as well, and binding metal ions vital for cell function would help maintain cellular nutritional needs. Transitional ion binding has been shown to be a key factor in regulating virulence factors of Staphylococcus aureus ${ }^{37}$ and may be similarly important in the regulation of venom molecules, or perhaps the altered host cells need the ability to regulate these molecules autonomously ${ }^{29}$. Metal ion binding also participates in buffering the effects of oxidative stress.

Cells are only so durable with a finite amount of processes they can carry out before they are no longer able to properly function ${ }^{38}$, similar to a machine with moving parts. Those parts must be well maintained to extend the length of time that machine lasts and how much work it can complete efficiently over its lifetime, just like oil changes and other scheduled maintenance and parts replacement allows a machine to operate efficiently and not break down. A cell is similar in that, as cellular output increases, so too do the wear and tear on the cellular mechanism. Oxidation-reduction reactions and catabolic pathways are essential for regulating cellular turnover by degrading worn-out cellular components at a rate fast enough to keep up with the increased cellular function, allowing for newly transcribed cellular components to replace the old. 
Maybe the most interesting protein found in fraction 5 was Toll-like venom protein, which is homologous to Drosophila Toll but with a smaller Leucine-rich repeat (LRR) domain1. This Toll-like venom protein may be essential for allowing the venosome to gain access to Drosophila hemocytes. Drosophila Toll-like receptors (TLR) are transmembrane proteins required for receiving signals, which are transduced to activate downstream NFKB transcription factors and immune signaling $11,39,40$. The Toll-like venom homolog has conserved LRRs but lacks transmembrane or TIR domains ${ }^{1}$. It would be worth exploring how altering the Toll-like venom receptor affects the venosomes ability to infiltrate Drosophila plasmatocytes, or if there is a change in immune response post-infection.

In other wasp models, the venom contains a diversity of venosome types with varying sizes and shapes. Although the function of these different types of venosomes has not fully been described to date, it would be interesting to explore the idea that G1 venom contains different types of venosomes, and if so, what different cargos and functions are associated with each specific venosome type. A next step could be to get an electronic microscopy scan of G1 venom to visually quantify the venosomes and determine if venosomes all look the same or if they differ in size and shape like in other wasp venoms. If there are other types of venosome, then maybe there are also other cell types that are targeted or can be targeted by the venosomes in G1 venom, or perhaps there are more specialized venosomes to more specifically regulate different aspects of the host's intracellular environment. By understanding the principle underlying the function of venosomes, researchers may gain insight into potential therapeutic applications in medicines. Utilizing viral vectors has been a powerful tool in the application of gene therapy. Venosomes' ability to transport cell restructuring cargo to specific cell types could lend to current gene therapy methods used today. 
The long-term goal of this research is to apply this understanding towards diseases caused by inhibited Ca2+ cycling, such as heart failure, and towards novel tissue-specific therapeutics to target diseases ${ }^{4,22,41,42}$ such as cancers that show dysregulated cell signaling. The data collected on G1 venom properties to date are a good starting point for future research into understanding the biogenesis of venosomes, possibly contributing to the emerging field of exosome-mediated therapies ${ }^{22,42}$; and understanding cell altering venosomes and the function of virulent proteins may be a window into effectively manipulating cell production and lifespan. With improvements and modifications to the venom protein purification process, and several more replicates of sucrose gradients, the process of generating venom fractions that dependably separate venom components into consistent layers can be improved. An improved purification protocol would reduce variability in samples, and increase assay accuracy. The research completed so far is an important step towards understanding venosomes and mapping G1 venom protein function. 


\section{CHAPTER IV: MATERIALS AND METHODS}

\section{Biological Material}

The Ganaspis hookeri (strain G1) wasps were caught in Homestead, Florida in 2008. All parasitoids were reared at about $25^{\circ} \mathrm{C}$ on Drosophila yakuba. After emergence, adults were kept

at $25^{\circ} \mathrm{C}$ on agar medium with honey on the cotton tube stopper. D. melanogaster (w1118 strain) were obtained from the Bloomington Drosophila Stock Center (Bloomington, IN). The D. yakuba R strain was obtained from the San Diego Species Center (San Diego, CA). All Drosophila were reared on a standard medium (10\% cornmeal, $10 \%$ yeast, agar, and nipagine) at $25^{\circ} \mathrm{C}$.

\section{Wasp Infections}

Wasp infections were performed as described ${ }^{1-4}$. Three female wasps were allowed to attack second instar fly larvae in $5 \mathrm{~mL}$ plastic vials, filled with Drosophila medium at $25^{\circ} \mathrm{C}$.

\section{Venom Protein Isolation}

50-100 female wasps were dissected into 100 ul 1x PBS, augmented with $0.37 \mathrm{mg}$ of Complete Mini Protease Inhibitor Cocktail (Roche). Venom gland tissues are collected on ice, then manually homogenized in non-lysing conditions. Then the cells are pelleted by centrifugation at 10,000 $\mathrm{g}$ for 5 minutes, and the venom supernatant is removed and stored in a 1.5mL Eppendorf tube at $4^{\circ} \mathrm{C}$.

\section{Venom Protein Purification}

The $10-50 \%$ sucrose gradient is underlaid in a $600600 \mathrm{uL}$ tube. $100 \mathrm{uL}$ of venom isolate is added to the sucrose gradient ${ }^{43}$. The gradient is centrifuged at $130,000 \operatorname{rcf}$ for 12 hours at $4^{\circ} \mathrm{C}$. Five equal fractions are removed and placed in separate 1.5mL Eppendorf tubes. 


\section{Venom Sonification}

In order to disrupt the venosome membrane and ensure proteins potentially held as cargo within venosomes are released, each fraction of purified venom samples is sonicated. From each fraction, a 1:1 ratio of purified venom samples are then placed in a sonicating water bath for 20 minutes at room temperature. Next, the samples are centrifuged at 10,000g for 10 minutes at $4^{\circ} \mathrm{C}$. Then, $10 \mu \mathrm{l}$ of DTT for every $40 \mu \mathrm{l}$ of $2 \mathrm{x}$ sample buffer is added and incubated for 10 minutes at $70^{\circ} \mathrm{C}^{43}$.

\section{SDS-PAGE Gel Electrophoresis}

$30 \mathrm{ul}$ of each of the 5 sonicated sucrose gradient fractions is applied in an individual well of 7.5\% polyacrylamide SDS-PAGE agarose gel, then ran at $40 \mathrm{~mA}$ per gel for $\sim 50$ minutes, or when the dye runs off the gel.

\section{Protein Stain}

Following SDS-PAGE, the gel is incubated in Gold Biotechnology Blazin' Bright luminescent UV protein-dye solution for 5 minutes. The UV signal will show stained bands where proteins are present and will be visualized with a digital camera and accompanying software

\section{Western Blot}

The proteins are transferred from the SDS-PAGE gel to a PVDF membrane. After the transfer, incubate the membrane in milk block (1x Tris-Buffered Saline (TBS) $+5 \%$ powder milk $+2 \%$ bovine serum albumin) for 1 hour to reduce non-specific binding. Wash three times in TBST (1X TBS + 0.2\% Tween 20). Then, incubated with the primary antibody in milk block (.1 $\mathrm{ml}$ anti-rabbit SERCA $+.9 \mathrm{ml}$ milk block), and secondary antibody $(.1 \mathrm{ml}$ anti-rabbit $\mathrm{HRP}+.9$ $\mathrm{ml}$ milk block). The secondary antibody aids in the detection of proteins by binding to the 
primary antibody. The secondary antibody is incubated with an enhanced chemiluminescent (ECL) to produce a signal that is visualized with a digital camera and accompanying software.

\section{Sample Preparation for MS Analysis}

Thirty-five microliters each of sucrose gradient fractions in SDS sample buffer (2\% SDS, 10\% glycerol, $50 \mathrm{mM}$ Tris, 0.5X PBS, trace bromophenol blue) were separated onto a NuPAGETM 10\% Bis-Tris Protein gel (Cat.\# NP0315BOX, Invitrogen) at 200V constant for 10min. The gel was stained using a Colloidal Blue Staining Kit (Cat.\#LC6025, Invitrogen) following the manufacturer's instruction.

Each gel lane was excised and digested overnight at $37^{\circ} \mathrm{C}$ with Pierce ${ }^{\mathrm{TM}}$ Trypsin Protease, MS Grade (Cat.\#90058, Thermo Scientific) as per manufacturer’s instruction. Digests were reconstituted in 0.1\%FA in 5:95 ACN:ddH2O at $~ 0.1 \mathrm{ug} / \mathrm{uL}$.

\section{Mass Spec. nLC-ESI-MS2 Analysis and Database Searches}

Peptide digests (8 $8 \mathrm{~L}$ each) were injected onto a 1260 Infinity nHPLC stack (Agilent 11 Technologies), and separated using a 100 micron I.D. x $12.5 \mathrm{~cm}$ pulled tip C-18 column (Jupiter C-18 $300 \AA$, 5 microns, Phenomenex). This system runs in-line with a Thermo Orbitrap Velos Pro-hybrid mass spectrometer, equipped with a nano-electrospray source (Thermo Fisher Scientific), and all data were collected in CID mode. The nHPLC was configured with binary mobile phases that included solvent A (0.1\% FA in ddH2O), and solvent B ( $0.1 \%$ FA in $15 \%$ ddH2O / 85\% ACN), programmed as follows; 10min @ 5\%B (2 $\mu \mathrm{L} / \mathrm{min}$, load), 90min @ 5\% -

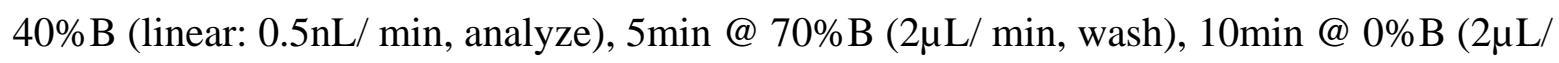
min, equilibrate). Following each parent ion scan (300 -1200m/z @ 60k resolution), fragmentation data (MS2) was collected on the topmost intense 15 ions. For data-dependent scans, charge state screening and dynamic exclusion were enabled with a repeat count of 2 , 
repeat duration of the 30 s, and exclusion duration of the 90 s

The XCalibur RAW files were collected in profile mode, centroided and converted to MzXMLusing ReAdW v. 3.5.1. The data were searched using G1 protein sequences and SEQUEST, which was set for two maximum missed cleavages, a precursor mass window of 20ppm, trypsin digestion, variable modification C @ 57.0293, and M @ 15.9949.

\section{Mass Spec. Peptide Filtering, Grouping, and Quantification}

The list of peptide IDs generated based on SEQUEST (Thermo Fisher Scientific) search results were filtered using Scaffold (Protein Sciences, Portland Orgegon). Scaffold filters and groups all peptides to generate and retain only high confidence IDs while also generating

normalized spectral counts (N-SC’s) across all samples for the purpose of relative quantification. The filter cut-off values were set with minimum peptide length of $>5$ AA's, with no MH+1 charge states, with peptide probabilities of $>80 \%$ C.I., and an FDR $<1.0 \%$. Scaffold incorporates the two most common methods for statistical validation of large proteome datasets, the false discovery rate (FDR) and protein probability [17-19]. Relative quantification across experiments was then performed via spectral counting [20, 21], and when relevant, spectral count abundances were then normalized between samples [22]. Using quantitative analysis, I determined that fraction 5 contained the most vSERCA and would be the fraction I focused on.

\section{Ex vivo SERCA Activity Assay}

Healthy third instar larvae are washed in 1x PBS and are dissected into wells of a diagnostic slide (Tekdon, Inc.) containing 30 $\mu \mathrm{L}$ of 1x PBS. Three wells are used with 5 larvae bled into each well for at least 5 minutes each to allow hemocytes time to adhere to the slide. The PBS is removed and either experimental buffer (Fluo-4 + venom fraction) or control buffer (Fluo-4 + PBS containing an amount of sucrose equivalent to the concentration found in the 
venom fraction) is added. Fluo-4 AM from Thermo Fisher Scientific is a calcium indicator that increases in fluorescence upon binding Ca2+. Samples were allowed to incubate in the dark for 15 minutes before being placed in an Invitrogen Tali Image-Based Cytometer, where changes in cell fluorescence could be measured. The fluorescence intensity of cells is proportional to intracellular Ca2+ levels.

\section{Statistics}

All analyses were performed in RStudio version 1.1.463. Using the "multcomp" 4 , "Ime4” 45 , "ImerTest”46, "effects”47, "dplyr”48 packages. Mixed linear models, including replicate as a random effect, were used to test for differences of fluo-4 fluorescence levels between venom fraction and the control replicate groups. The model treats a replicate as a random effect while averaging the result by taking differences between replicates into account. The resulting whisker graph shows the distribution of fluorescent intensity data through quartiles. This disregards random effects in the final statistics.

\section{Immunohistochemistry}

5-10 venom glands were dissects into wells of a diagnostic slide containing 30 $\mu \mathrm{L}$ PBS. The PBS was replaced with 2\% formaldehyde in PBS and left to fix for 15-20 minutes. The tissues were washed with PBS at least 4 times and washed once with PBT (39.6 ml PBS + $400 \mathrm{ul}$ $20 \%$ Triton). The venom glands were then transferred to a $1.5 \mathrm{ml}$ Eppendorf tube and allowed to gently rock for one hour in a block solution of PBTG (9.9 ml PBT + $100 \mathrm{ul} \mathrm{4 \%} \mathrm{goat} \mathrm{serum).}$ Then, the block was replaced with SERCA Alexa 488 diluted at 1:1000 in PBTG as the primary antibody probe and allowed to incubate on a rocker at room temp for $5 \mathrm{hrs}$, or at $4^{\circ} \mathrm{C}$ overnight. The sample was washed 3-4 times with PBTG block for a total of at least 1 hour. The phalloidin 633, a green fluorescent dye that stains actin, is diluted at 1:1000 in PBGT as the secondary 
antibody probe and allowed to incubate on a rocker at room temp for $5 \mathrm{hrs}$ or at $4^{\circ} \mathrm{C}$ overnight wrapped in foil, then washed 3-4 times with PBTG and let the sample sit in last wash wrapped in foil until mounted. The samples were then viewed with a Leica SP8 confocal microscope.

\section{Bioinformatics}

The venom proteins identified by MS as co-localizing in fraction 5 with vSERCA were assembled into a FASTA sequence database. Because the G1 genome is not well annotated, BLAST was used to identify the homologous proteins in D. melanogaster, a species with a highly annotated genome. I used the command line BLAST (version 2.7.1) with a custom made BLAST database including all identified protein sequences from the D. melanogaster genome (from http://flybase.org, genome release 6.31).

The Flybase36 gene IDs were then used for gene ontology (GO) term mapping through Panther (http://geneontology.org/) ${ }^{49,50}$. The biological process, molecular function, and cellular component databases were individually searched and the D. melanogaster genome was used as the background gene set for enrichment analysis. GO terms with a corrected enrichment p-value $<0.05$ are considered significantly enriched in the sample. 
Fig. 1 Sucrose Gradient

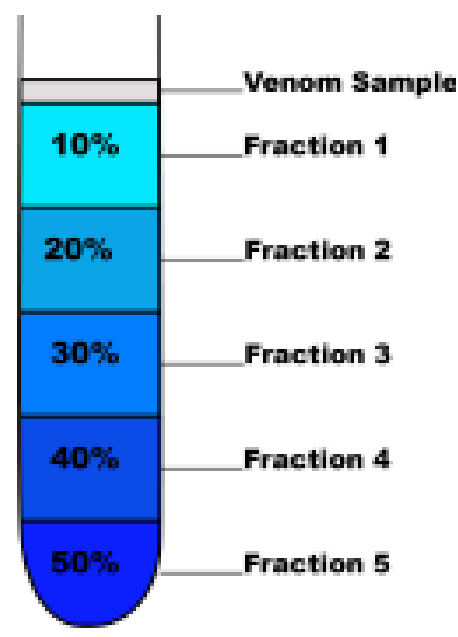

The Sucrose Gradient A 10-50\% sucrose gradient is established and after centrifugation at 130,000 ret for 12 hours at $4^{\circ} \mathrm{C}$, five equal layers are separated into individual $1.5 \mathrm{~mL}$ Eppendorf tubes as corresponding fractions.

Fraction 1 At a concentration of $10 \%$ sucrose and $90 \%$ PBS, this is the least dense layer of the gradient. This layer is expected to have individual venom proteins and peptides, as well as proteins and peptides that may have initially been a part of complexes that were disrupted during the purification or centrifugation processes.

Fraction 2 At a concentration of $20 \%$ sucrose, this layer is expected to have some overlapping venom proteins and peptides from fraction 1 and possibly less dense protein complexes.

Fraction 3 At a concentration of $30 \%$ sucrose, this this is where l'd expect to start seeing vensomes and other large protein complexes.

Fraction $4 \mathrm{At}$ a concentration of $40 \%$ sucrose this layer is expected to have venosomes and other large complexes that are overlapping and possibly more dense than fraction 3 .

Fraction 5 At a $1: 1$ concentration of sucrose to PBS this layer is the densest of the gradient. I would expect to see some overlapping venosomes and complexes from fraction 4 along with the most dense venosomes and venom protein complexes. However, I would not expect to see any pelleting because most of the venom content is expected to have reached its equilibrium point before reaching the bottom of the centrifuge tube. 
Fig. 2 Protein Gel and Western Blots
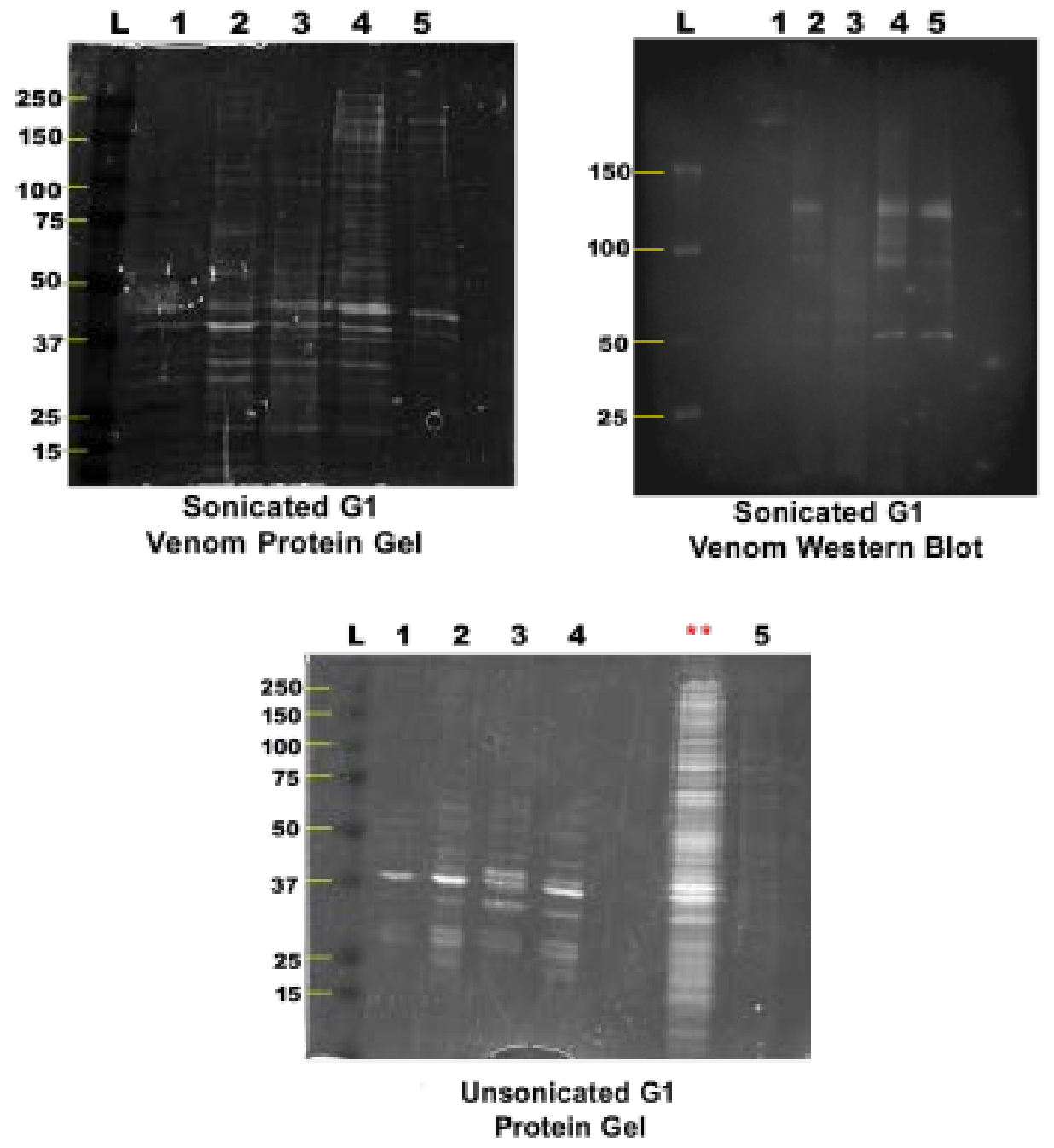

Figure 2. SDS-PAGE protein analysis of fractionated $G 1$ venom. For each panel, lane $L$ represents the ladder and is labeled by size. Each lane contains the corresponding sucrose venom fraction (ie. lane 1 contains fraction 1). (a) SDS-PAGE of unsonicated purified venom stained with Blazin' Bright UV protein stain. The brighter the signal, the more protein that was detected. The lane marked "* is a contaminated sample that was not analyzed further. (b) SDS-PAGE of sonicated purified venom stained with Blazin' Bright UV protein stain. (c) The same samples as B were analyzed by Western blot probed with anti-SERCA. 
Fig. 3 Venom Fractions 1-5 Calcium Assay Statistic Plots

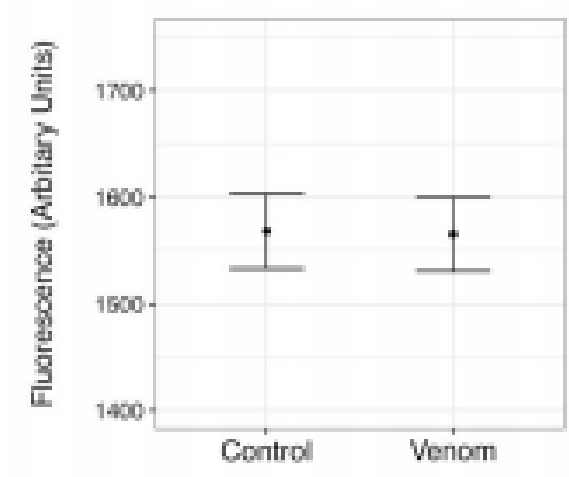

Fraction 1

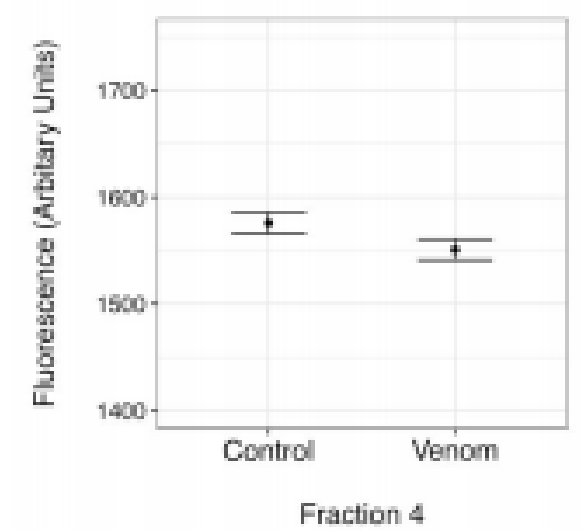

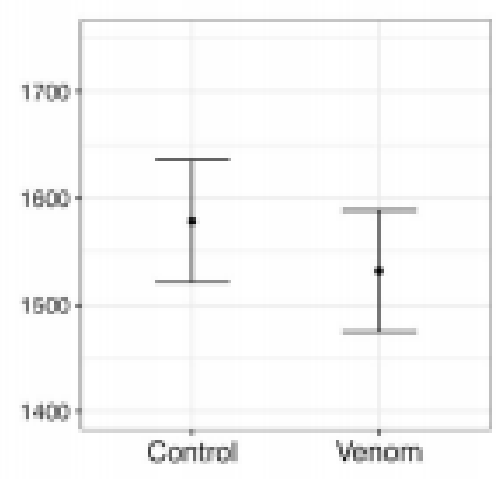

Fraction 2

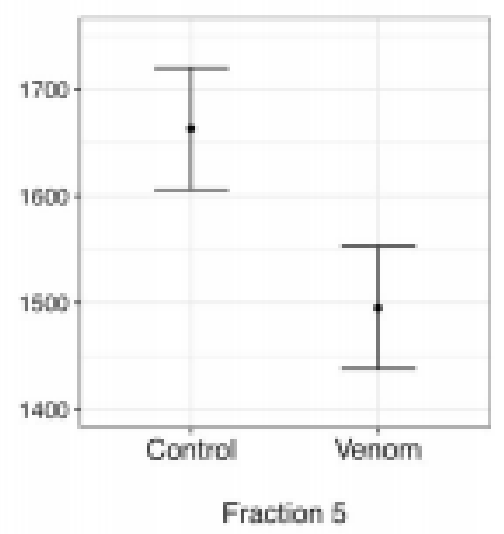

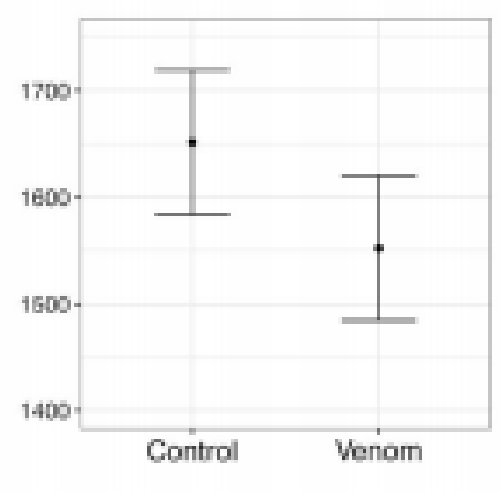

Fraction 3

Invitrogen Tali Image-Based Cytometer was used 10 measure the change in fluorescent intensity of Drosophila second instar larvae hemolymph samples that were incubated with calcium indicator Fluo-4 AM from Thermo Fisher Scientific. A linear modeling code was used in $R$ to gener ate the whisker graphs that reflect the change in fluorescent intensity. The graphs show the fluorescent intensity + - the standard error. 
Fig. 4 G1 Venom Apparatus Confocal Images

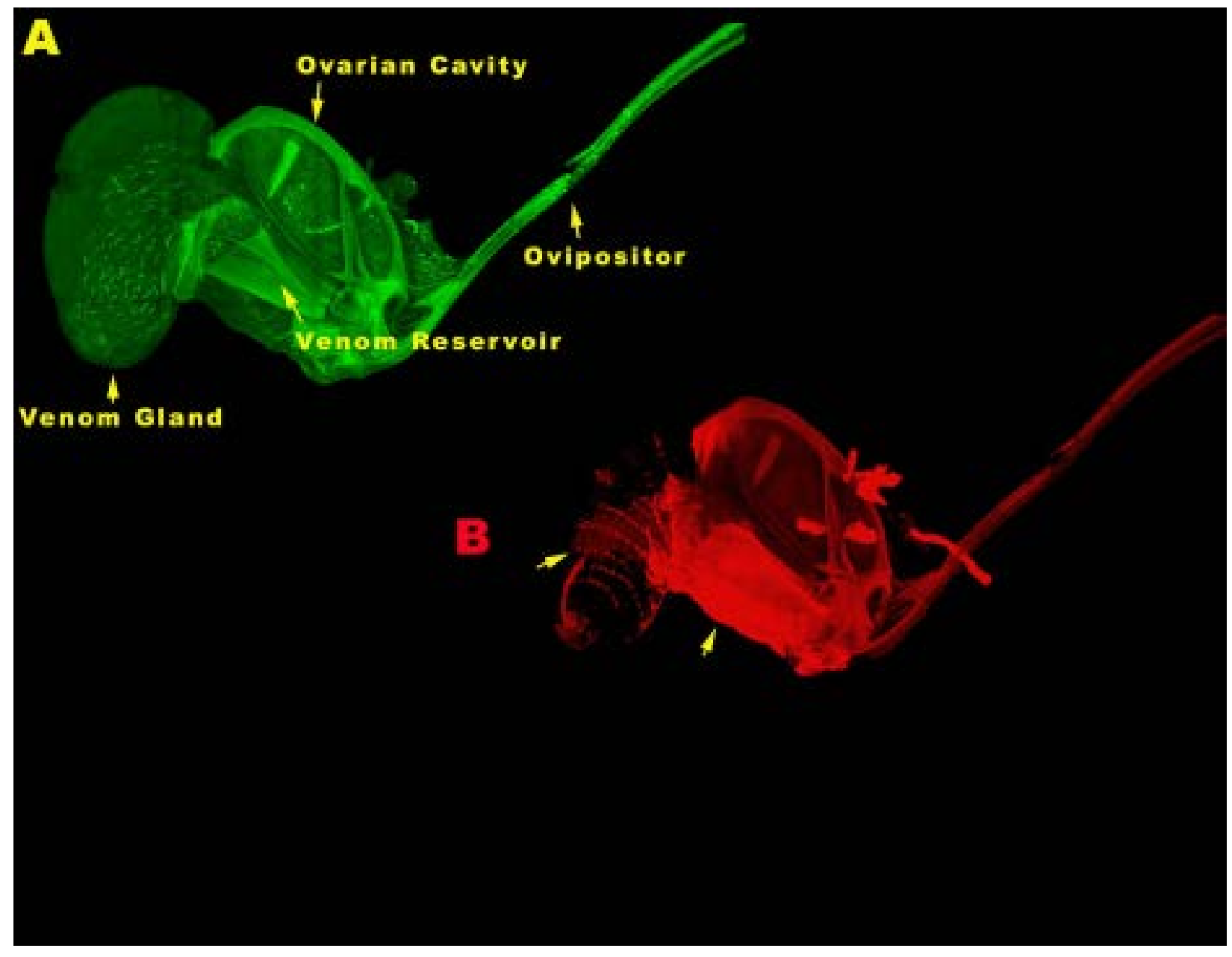

Figure 4. Confocal imaging of SERCA localization in the venom apparatus. Images were taken simultaneously using a 10x objective and 3.99 digital zoom. (A) Image of the whole G1 venom apparatus stained with anti-SERCA, and with structures labeled. (B) Image of the whole G1 venom apparatus labelled with phalloidin. 
Fig. 5 G1 Venom Gland Confocal Images

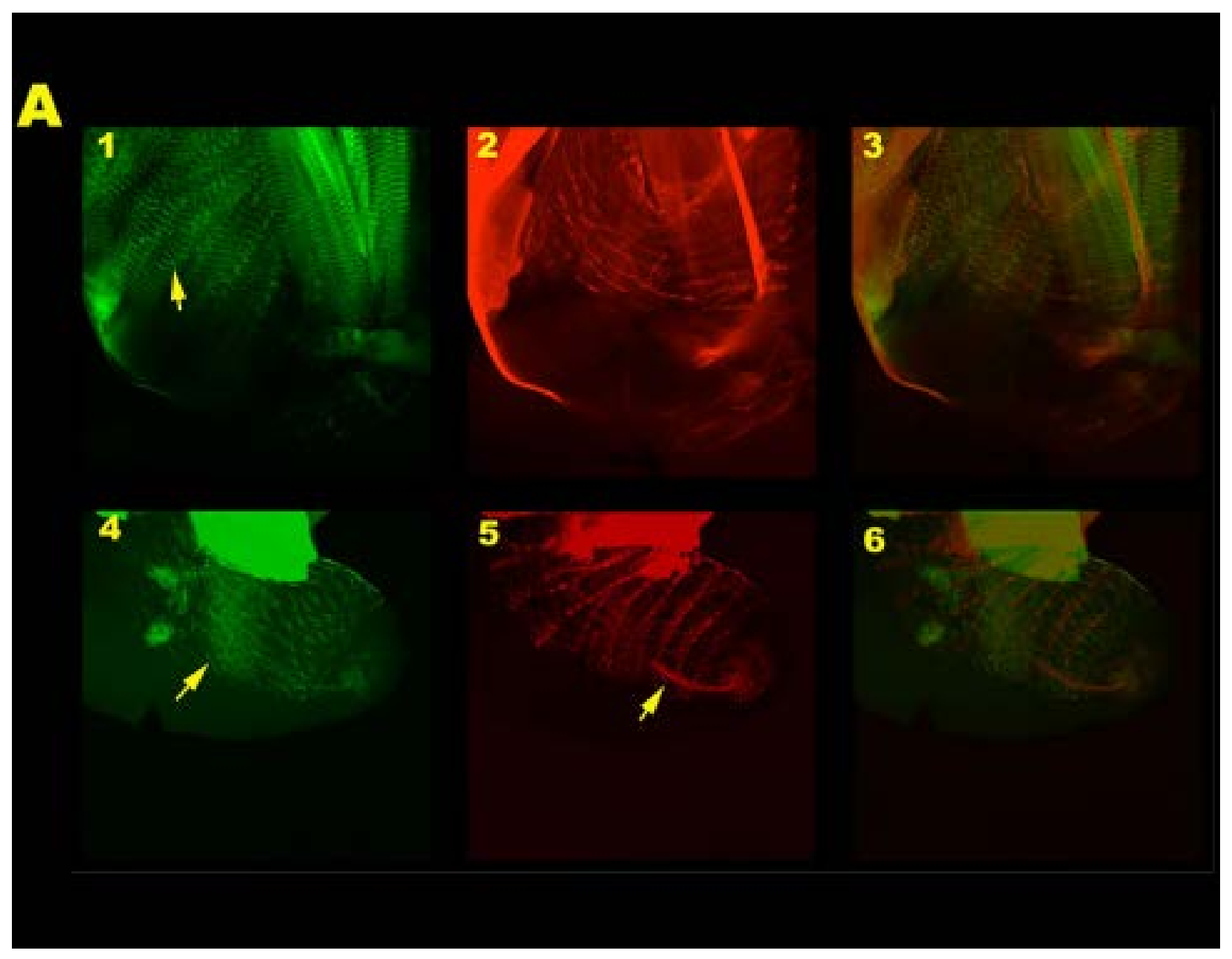

Figure 5. Confocal imaging of SERCA localization in the venom reservoir. Images taken using a 10x objective and 3.99 digital zoom of anti-SERCA (green) localized in the sarcoplasmic reticulum (A1), actin structures labeled with phalloidin (red) on the venom reservoir (A2), and a composite of the two images (A3). (A4-C6) Images taken using a 10x objective and 2.77 digital zoom of anti-SERCA (green) localized in the venom gland and venom reservoir (reservoir is indicated by the arrow) (A4), the winding system of tunnel-like actin structures (labelled with phalloidin, red) with the arrow indicating the secretory duct (A5) and a composite of the two images (A6). 
Table 1 Mass Spectrometry Fraction Quantification

\begin{tabular}{|c|c|}
\hline \multicolumn{2}{|c|}{\begin{tabular}{c} 
Mass Spectrometry Fraction Quantification \\
\hline Fraction
\end{tabular}} \\
\hline 1 & t of Proteins Found in Fraction \\
\hline 2 & 230 \\
\hline 3 & 311 \\
\hline 4 & 314 \\
\hline 5 & 320 \\
\hline Total & 291 \\
\hline
\end{tabular}


Table 2 Mass Spectrometry Analysis Fraction 5 Preview

\begin{tabular}{|c|c|c|}
\hline \multicolumn{3}{|c|}{$\begin{array}{c}\text { Mass Spectrometry Analysis } \\
\text { Fraction } 5 \text { Preview }\end{array}$} \\
\hline Accession ID & Flybase ID & Gene Symbol \\
\hline comp1045 & FBgn0263006 & SERCA \\
\hline comp1567 & FBgn0028325 & Pdha \\
\hline comp4522 & FBgn0035371 & AhcyL1 \\
\hline comp288 & FBgn0030449 & Fer3HCH \\
\hline comp3360 & $\mathrm{FBgn} 0261458$ & capt \\
\hline comp16783 & FBgn0022709 & Adk1 \\
\hline comp2164 & FBgn0040064 & yip2 \\
\hline comp4656 & FBgn0003255 & rk \\
\hline comp45454 & FBgn0264695 & Mhe \\
\hline comp3177 & $\mathrm{FBgn0035917}$ & Zasp66 \\
\hline comp12432 & $\mathrm{FBgn0010482}$ & К(2)01289 \\
\hline comp6889 & FBgn0034476 & Toll-7 \\
\hline comp882 & $\mathrm{FBgn0278608}$ & Dsp1 \\
\hline comp2103 & $\mathrm{FBgn0023023}$ & CAMP \\
\hline comp1291 & FBgn0028685 & Rpt4 \\
\hline comp303 & FBgn0026415 & Idgf4 \\
\hline
\end{tabular}


Table 3 Biological Process GO Terms

\begin{tabular}{|l|l|l|}
\hline \multicolumn{1}{|c|}{ GO ID } & \multicolumn{1}{|c|}{ Biological Process } \\
\hline GO:0051238 & sequestering of metal ion & P-Value \\
\hline GO:0010499 & $\begin{array}{l}\text { proteasomal ubiquitin- } \\
\text { independent protein catabolic } \\
\text { process }\end{array}$ & $1.03 E-04$ \\
\hline GO:0008063 & Toll signaling pathway & $8.77 E-04$ \\
\hline GO:0009063 & $\begin{array}{l}\text { cellular amino acid catabolic } \\
\text { process }\end{array}$ & $1.38 E-02$ \\
\hline GO:0007623 & circadian rhythm & $6.23 E-03$ \\
\hline GO:0048511 & rhythmic process & $6.08 E-03$ \\
\hline GO:0055114 & oxidation-reduction process & $1.33 E-06$ \\
\hline GO:0007166 & $\begin{array}{l}\text { cell surface receptor signaling } \\
\text { pathway }\end{array}$ & $4.99 E-02$ \\
\hline GO:0032989 & $\begin{array}{l}\text { cellular component } \\
\text { morphogenesis }\end{array}$ & $3.79 E-02$ \\
\hline GO:0044238 & primary metabolic process & $1.04 E-02$ \\
\hline
\end{tabular}


Table 4 Cell Composition GO Terms

\begin{tabular}{|l|r|r|}
\hline \multicolumn{1}{|c|}{ GO ID } & \multicolumn{1}{|c|}{ Cell Composition GO Terms } \\
\hline GO:0008043 & intracellular ferritin complex & P-Value \\
\hline GO:0032982 & myosin filament & $9.24 \mathrm{E}-03$ \\
\hline GO:0005839 & proteasome core complex & $1.78 \mathrm{E}-02$ \\
\hline GO:0030018 & Z disc & $1.76 \mathrm{E}-05$ \\
\hline GO:0015629 & actin cytoskeleton & $4.35 \mathrm{E}-02$ \\
\hline GO:0043233 & organelle lumen & $1.10 \mathrm{E}-03$ \\
\hline GO:0031974 & membrane-enclosed lumen & $4.29 \mathrm{E}-02$ \\
\hline
\end{tabular}


Table 5 Molecular Function GO Terms

\begin{tabular}{|c|c|c|}
\hline \multicolumn{3}{|c|}{ Molecular Function GO Terms } \\
\hline GO ID & Molecular Function & P-Value \\
\hline GO:0004030 & $\begin{array}{l}\text { aldehyde dehydrogenase } \\
{[\mathrm{NAD}(\mathrm{P})+] \text { activity }}\end{array}$ & $3.52 E-02$ \\
\hline GO:0008199 & ferric iron binding & 3.06E-02 \\
\hline GO:0051371 & muscle alpha-actinin binding & 3.69E-03 \\
\hline GO:0016620 & $\begin{array}{l}\text { oxidoreductase activity, acting } \\
\text { on the aldehyde or oxo group of } \\
\text { donors, NAD or NADP as } \\
\text { acceptor }\end{array}$ & 5.44E-03 \\
\hline GO:0005524 & ATP binding & $1.27 \mathrm{E}-02$ \\
\hline GO:0000166 & nucleotide binding & 7.01E-03 \\
\hline GO:0036094 & small molecule binding & 1.67E-02 \\
\hline GO:0003824 & catalytic activity & $1.20 E-05$ \\
\hline
\end{tabular}




\section{REFERENCES}

1. Alvarado, G. et al. Bioinformatic analysis suggests potential mechanisms underlying parasitoid venom evolution and function. Genomics 112, 1096-1104 (2020).

2. Ferrarese, R., Morales, J., Fimiarz, D., Webb, B. A. \& Govind, S. A supracellular system of actin-lined canals controls biogenesis and release of virulence factors in parasitoid venom glands. J. Exp. Biol. 212, 2261-2268 (2009).

3. Konno, K., Kazuma, K. \& Nihei, K. Peptide Toxins in Solitary Wasp Venoms. Toxins 8, 114 (2016).

4. Moreno, M. \& Giralt, E. Three Valuable Peptides from Bee and Wasp Venoms for Therapeutic and Biotechnological Use: Melittin, Apamin and Mastoparan. Toxins 7, 1126-1150 (2015).

5. Wan, B. et al. Venom Atypical Extracellular Vesicles as Interspecies Vehicles of Virulence Factors Involved in Host Specificity: The Case of a Drosophila Parasitoid Wasp. Front. Immunol. 10, 1688 (2019).

6. Er, A., Sak, O., Ergin, E., Uçkan, F. \& Rivers, D. B. Venom-Induced Immunosuppression: An Overview of Hemocyte-Mediated Responses. Psyche J. Entomol. 2011, 1-14 (2011).

7. Mortimer, N. T. Parasitoid wasp virulence: A window into fly immunity. Fly (Austin) 7, 242-248 (2013).

8. Mortimer, N. T. et al. Parasitoid wasp venom SERCA regulates Drosophila calcium levels and inhibits cellular immunity. Proc. Natl. Acad. Sci. U. S. A. 110, 9427-9432 (2013). 
9. Goecks, J. et al. Integrative approach reveals composition of endoparasitoid wasp venoms. PloS One 8, e64125 (2013).

10. Kim-Jo, C., Gatti, J.-L. \& Poirié, M. Drosophila Cellular Immunity Against Parasitoid Wasps: A Complex and Time-Dependent Process. Front. Physiol. 10, 603 (2019).

11. Nakamoto, M. et al. Virus recognition by Toll-7 activates antiviral autophagy in Drosophila. Immunity 36, 658-667 (2012)

12. Davies, S. A. et al. Cell signalling mechanisms for insect stress tolerance. J. Exp. Biol. 217, 119-128 (2014).

13. Rizki, R. M. \& Rizki, T. M. Selective destruction of a host blood cell type by a parasitoid wasp. Proc. Natl. Acad. Sci. U. S. A. 81, 6154-6158 (1984).

14. Clapham, D. E. Calcium Signaling. Cell 131, 1047-1058 (2007).

15. Vig, M. \& Kinet, J.-P. Calcium signaling in immune cells. Nat. Immunol. 10, 21-27 (2009).

16. Lamboley, C. R., Murphy, R. M., McKenna, M. J. \& Lamb, G. D. Sarcoplasmic reticulum $\mathrm{Ca}^{2+}$ uptake and leak properties, and SERCA isoform expression, in type I and type II fibres of human skeletal muscle: SR $\mathrm{Ca}^{2+}$ uptake in human skeletal muscle fibres. J. Physiol. 592, 1381-1395 (2014).

17. Park, W. J. \& Oh, J. G. SERCA2a: a prime target for modulation of cardiac contractility during heart failure. BMB Rep. 46, 237-243 (2013).

18. Periasamy, M. et al. Role of SERCA Pump in Muscle Thermogenesis and Metabolism. Compr. Physiol. 7, 879-890 (2017). 
19. Sanyal, S., Jennings, T., Dowse, H. \& Ramaswami, M. Conditional mutations in SERCA, the Sarco-endoplasmic reticulum Ca2+-ATPase, alter heart rate and rhythmicity in Drosophila. J. Comp. Physiol. [B] 176, 253-263 (2006).

20. Akers, J. C., Gonda, D., Kim, R., Carter, B. S. \& Chen, C. C. Biogenesis of extracellular vesicles (EV): exosomes, microvesicles, retrovirus-like vesicles, and apoptotic bodies. $J$. Neurooncol. 113, 1-11 (2013).

21. Alenquer, M. \& Amorim, M. J. Exosome Biogenesis, Regulation, and Function in Viral Infection. Viruses 7, 5066-5083 (2015).

22. Vlassov, A. V., Magdaleno, S., Setterquist, R. \& Conrad, R. Exosomes: current knowledge of their composition, biological functions, and diagnostic and therapeutic potentials. Biochim. Biophys. Acta 1820, 940-948 (2012).

23. Ronquist, K. G. et al. Energy-requiring uptake of prostasomes and PC3 cell-derived exosomes into non-malignant and malignant cells. J. Extracell. Vesicles 5, 29877 (2016).

24. Hagen, B. M., Boyman, L., Kao, J. P. Y. \& Lederer, W. J. A comparative assessment of fluo Ca2+ indicators in rat ventricular myocytes. Cell Calcium 52, 170-181 (2012).

25. Endo, M. Calcium release from the sarcoplasmic reticulum. Physiol. Rev. 57, 71-108 (1977).

26. Kaur, J. \& Debnath, J. Autophagy at the crossroads of catabolism and anabolism. Nat. Rev. Mol. Cell Biol. 16, 461-472 (2015).

27. Collins, G. A. \& Goldberg, A. L. The Logic of the 26S Proteasome. Cell 169, 792-806 (2017).

28. Bard, J. A. M. et al. Structure and Function of the 26S Proteasome. Annu. Rev. Biochem. 87, 697-724 (2018). 
29. Wang, J. \& Pantopoulos, K. Regulation of cellular iron metabolism. Biochem. J. 434, 365-381 (2011).

30. Smith, S. J. \& Saggerson, E. D. Regulation of pyruvate dehydrogenase activity in rat epididymal fat-pads and isolated adipocytes by adrenaline. Biochem. J. 174, 119-130 (1978).

31. Stansbie, D., Brownsey, R. W., Crettaz, M. \& Denton, R. Acute effects in vivo of antiinsulin serum on rates of fatty acid synthesis and activities of acetyl-coenzyme A carboxylase and pyruvate dehydrogenase in liver and epididymal adipose tissue of fed rats. Biochem. J. 160, 413-416 (1976).

32. Bunnag, P. et al. Impaired in vivo adrenergic responses in diet-induced hypertensive rats. Hypertens. Res. 20, 17-21 (1997).

33. Huber, S. C. \& Huber, J. L. Role of sucrose-phosphate synthase in sucrose metabolism in leaves. Plant Physiol. 99, 1275-1278 (1992).

34. Pontis, H. G. The role of sucrose and fructosylsucrose in fructosan metabolism. Physiol. Plant. 23, 1089-1100 (1970).

35. Ruan, Y.-L. Signaling role of sucrose metabolism in development. Mol. Plant 5, 763-765 (2012).

36. FlyBase Homepage. http://flybase.org/.

37. Cassat, J. E. \& Skaar, E. P. Metal ion acquisition in Staphylococcus aureus: overcoming nutritional immunity. Semin. Immunopathol. 34, 215-235 (2012).

38. Yang, J. et al. Cell size and growth rate are major determinants of replicative lifespan. Cell Cycle 10, 144-155 (2011). 
39. Anthoney, N., Foldi, I. \& Hidalgo, A. Toll and Toll-like receptor signalling in development. Development 145, (2018).

40. Janssens, S. \& Beyaert, R. Role of Toll-like receptors in pathogen recognition. Clin. Microbiol. Rev. 16, 637-646 (2003).

41. Branski, L. K., Gauglitz, G. G., Herndon, D. N. \& Jeschke, M. G. A REVIEW OF GENE AND STEM CELL THERAPY IN CUTANEOUS WOUND HEALING. Burns J. Int. Soc. Burn Inj. 35, 171-180 (2009).

42. Tricarico, C., Clancy, J. \& D’Souza-Schorey, C. Biology and biogenesis of shed microvesicles. Small GTPases 8, 220-232 (2016).

43. Greening, D. W., Xu, R., Ji, H., Tauro, B. J. \& Simpson, R. J. A protocol for exosome isolation and characterization: evaluation of ultracentrifugation, density-gradient separation, and immunoaffinity capture methods. Methods Mol. Biol. Clifton NJ 1295, 179-209 (2015).

44. Bretz, F., Hothorn, T. \& Westfall, P. Multiple Comparison Procedures in Linear Models. in COMPSTAT 2008 (ed. Brito, P.) 423-431 (Physica-Verlag HD, 2008).

45. Bates, D. et al. Package 'Ime4’. Convergence 12, 2 (2015).

46. Kuznetsova, A., Brockhoff, P. B. \& Christensen, R. H. B. lmerTest package: tests in linear mixed effects models. J. Stat. Softw. 82, (2017).

47. Fox, J. et al. Package 'effects’. (2019).

48. Wickham, H. with dplyr. in (2014).

49. PANTHER - Gene List Analysis. http://pantherdb.org/webservices/go/overrep.jsp. 50. Gene Ontology Resource. Gene Ontology Resource http://geneontology.org/. 